\title{
Verfassungsgerichte in den EU-Staaten: Wahlverfahren, Kompetenzen und Organisationsprinzipien
}

Nach der Osterweiterung der Europäischen Union findet sich inzwischen in der Mehrzahl der EU-Mitgliedstaaten ein Verfassungsgericht - und der traditionelle Sonderfall wurde zum Normalfall. Verfassungsgerichte spielen im Alltag der Bürger eine wichtige Rolle und beeinflussen die politische Willensbildung und Entscheidung, da sie das Recht haben, durch das Parlament verabschiedete Normen außer Kraft zu setzen, wenn diese - nach der Interpretation des Gerichts nicht im Einklang mit der Verfassung stehen. Vor diesem Hintergrund wird Verfassungsgerichten nicht nur seitens der Rechtswissenschaft, sondern zunehmend auch von der Vergleichenden Politikforschung als „Vetospieler" ${ }^{\text {“1 }}$ oder „konsensdemokratische Elemente“ ${ }^{‘ 2}$ große Bedeutung zugemessen.

Im Gegensatz zu Parlamenten ist das politikwissenschaftliche Verständnis von Verfassungsgerichten aber eher oberflächlich. Die bisherige Forschung hat sich vor allem mit der allgemeinen Hypothese der zunehmenden Justizialisierung der Politik befasst, die von Landfried ${ }^{3}$ für das Bundesverfassungsgericht und von Stone $^{4}$ für den Conseil constitutionnel anhand der abstrakten Normenkontrolle entwickelt und dann auf anderen Länder übertragen wurde. Nur wenige neuere Beiträge weisen darauf hin, dass dieser „Verrechtlichung“ durch bestimmte institutionelle Regeln oder Akteurskonstellationen Grenzen gesetzt sind. ${ }^{5}$

1 Tsebelis, G.: Veto Players, Princeton, 2002.

2 Lijphart, A.: Patterns of Democracy, New Haven, CT, 1999.

3 Landfried, C.: Bundesverfassungsgericht und Gesetzgeber, Baden-Baden, 1984.

4 Stone, A.: The Birth of Judicial Politics in France, New York, 1992.

5 Vanberg, G.: Abstract Judicial Review, Legislative Bargaining, and Policy Compromise, in: Journal of Theoretical Politics, 10/3 (1998), 299-326; ders.: Verfassungsgerichtsbarkeit und Gesetzgebung. Zum politischen Spielraum des Bundesverfassungsgerichts, in: Ganghof, S./Manow, P. (Hg.): Mechanismen der Politik - Strategische Interaktion im deutschen Regierungssystem, Frankfurt/M., 2005, 183-214; Magalhães, P.C.: The Limits to Judicialization. Legislative Politics and Constitutional Review in the Iberian Democracies, PhD Dissertation, Ohio State University, 2003; Hönnige, C.: Verfassungsgericht, Regierung und Opposition, Wiesbaden, 2007. 
Die vorliegende Dokumentation gibt eine Übersicht über die wichtigsten institutionellen Merkmale von Verfassungsgerichten in den west- und osteuropäischen EU-Staaten, die für ihren Einfluss auf politische Entscheidungen von Bedeutung sind: Richterwahl und politische Unabhängigkeit, Zugangswege zum Verfassungsgericht sowie die internen Organisationsprinzipien. Im Ergebnis zeigt sich, dass diese institutionellen Regelungen im internationalen Vergleich beträchtlich variieren.

\section{Institutionelle Merkmale von Verfassungsgerichten in Europa}

Verfassungsgerichtsbarkeit lässt sich grundsätzlich in zwei Typen untergliedern: ein deutsch-österreichisches Modell (zentralisiertes System) und ein US-amerikanisches Modell (dezentralisiertes System). Das zentralisierte, von Hans Kelsen 1920 entworfene Modell unterscheidet sich erheblich von der Struktur der Verfassungsgerichtsbarkeit in den Common-law-Ländern, die dem US-amerikanischen Typus folgen. ${ }^{6}$ Seine Kennzeichen sind die Existenz eines gesonderten Verfassungsgerichtes, das vom regulären Instanzenzug isoliert ist und als einziges Gericht die Möglichkeit hat, konstitutionelle Normen aufzuheben. Üblicherweise verfügen diese Gerichte über ein breites Spektrum an Verfahrensarten mit jeweils verschiedenen Antragsberechtigten.

In den Mitgliedstaaten der Europäischen Union folgen alle Länder mit Verfassungsgericht, mit der Ausnahme Estlands sowie partiell Portugals, dem zentralisierten Modell (vgl. Tabelle 1). In Estland ist der Supreme Court in den regulären Instanzenzug eingebunden und besitzt eine Kammer, die sich explizit um verfassungsrechtliche Fragen kümmert. ${ }^{7}$ Der Fall Portugals beweist, dass die Grenzen zwischen den Modellen fließend sein können. Zwar kennt das Land ein spezialisiertes Verfassungsgericht mit einer institutionellen Gestaltung entsprechend dem Kelsen'schen Modell, bei der konkreten Normenkontrolle sind aber auch die regulären Prozessgerichte prüfungsberechtigt und müssen - von weni-

6 Cappelletti, M./Ritterspach T.: Die gerichtliche Kontrolle der Verfassungsmäßigkeit der Gesetze in rechtsvergleichender Betrachtung, in: Jahrbuch des öffentlichen Rechts, 20 (1971), 65-86, hier 81 ff.; Stone Sweet, A.: Constitutional Courts and Parliamentary Democracy, in: West European Politics, 25/1 (2002), 77-100, hier $79 \mathrm{f}$.

7 Schmidt, C.: Verfassungskontrolle in Estland, in: Hofmann, M./Küpper H. (Hg.): Kontinuität und Neubeginn. Staat und Recht in Europa zu Beginn des 21. Jahrhunderts, Baden-Baden, 2001, 471-483, hier 471; Schmitz, T.: Die Kontrolle der Verfassungsmäßigkeit von Gesetzen in den baltischen Staaten, in: ZSE, 1/4 (2003), 555-578. 
Tabelle 1: Mitgliedstaaten der Europäischen Union mit Verfassungsgericht

\begin{tabular}{|l|l|c|c|}
\hline Staat & Verfassungsgericht & Gründung & Tätigkeit seit \\
\hline Belgien & Cour d'Arbitrage & 1983 & 1984 \\
\hline Bulgarien & Konstitutzionen Sud & 1991 & 1991 \\
\hline Deutschland & Bundesverfassungsgericht & 1949 & 1951 \\
\hline Estland & Vabariigi Riigikohus & 1993 & 1993 \\
\hline Frankreich & Conseil constitutionnel & 1958 & 1959 \\
\hline Italien & Corte costituzionale & 1948 & 1956 \\
\hline Lettland & Satversmes tiesa & 1996 & 1996 \\
\hline Litauen & Konstitucinis Teismas & 1993 & 1997 \\
\hline Österreich & Verfassungsgerichtshof & $1920 / 1945$ & $1923 / 1946$ \\
\hline Polen & Trybunal Konstytucyjny & $1982 / 1997$ & $1985 / 1997$ \\
\hline Portugal & Tribunal Constitucional & 1982 & 1983 \\
\hline Rumänien & Curtea Constitutionala & 1992 & 1992 \\
\hline Spanien & Tribunal Constitucional & 1979 & 1980 \\
\hline Slowakei & Ústavný súd & 1993 & 1993 \\
\hline Slowenien & Ustavno sodišče & 1994 & 1994 \\
\hline
\end{tabular}

Quelle: Eigene Darstellung.

gen Ausnahmen abgesehen - das Verfahren an das Tribunal Constitucional weiter verweisen. ${ }^{8}$

Trotz dieser grundlegenden Gemeinsamkeit unterscheiden sich die Verfassungsgerichte in den EU-Staaten hinsichtlich ihrer institutionellen Ausgestaltung erheblich. Deshalb werden im Folgenden jene Regelungen näher betrachtet, die das Verhalten der politischen Akteure in besonderer Weise beeinflussen:

- Verfahren der Richterwahl: Hierbei sind die Regelungen, die die politische Auswahl der Richter steuern, von jenen zu unterscheiden, die formale Qualifikationsanforderungen festlegen.

- Isolation des Gerichts: Dazu zählen institutionelle Regeln, die die Richter oder das Gericht von politischem Druck fernhalten, also insbesondere Wiederwahl, Abwahl sowie die mögliche Vermeidung von Urteilen.

- Kompetenzausstattung: Hierbei liegt der Fokus auf jenen Verfahrensarten, die dazu genutzt werden können, um die Regierungspolitik zu beeinflussen. Dazu zählen insbesondere die abstrakte und konkrete Normenkontrolle, Kompetenzstreitigkeiten und Verfassungsbeschwerden.

8 Cardoso da Costa, J. M. M.: Die Verfassungsgerichtsbarkeit in Portugal, in: Starck, C./Weber, A. (Hg.): Verfassungsgerichtsbarkeit in Westeuropa, Baden-Baden, 1986, 297-307, hier 297. 
- Binnenorganisation und Entscheidungsregeln: Sie stellen das strategische Umfeld dar, in dem Regierung, Opposition und andere Kläger versuchen ihre Interessen durchzusetzen. Von besonderer Bedeutung ist dabei, ob das Plenum, Senate und Kammern über einen Fall entscheiden, wie der Berichterstatter ausgewählt wird, nach welcher Mehrheit das Gericht entscheidet, ob der Präsident ein Stichvotum hat und ob ein Richter seine abweichende Meinung zu Protokoll geben kann bzw. das individuelle Abstimmungsergebnis veröffentlicht wird.

\section{Die Auswahl der Verfassungsrichter}

Die Regelungen zur Auswahl der Verfassungsrichter und ihrer Amtszeit sind in normativer und analytischer Hinsicht von Belang. Aus normativer Sicht konstituieren politische und berufliche Selektionskriterien ex ante die Legitimität eines Gerichts: Wie bereits Kelsen feststellte, ist die politische Auswahl der elektorale Bezug, den das Gericht zur Bevölkerung hat. Sie muss begründen, weshalb eine kleine Gruppe von Juristen das Recht erhält, Entscheidungen von enormer politischer Tragweite zu fällen. Hohe berufliche Auswahlstandards können vermeiden, dass das Gericht an Legitimität verliert, indem Urteile offensichtlich schlecht begründet oder nach politischen Wertvorstellungen der Richter gefällt werden. Deshalb werden entsprechende Qualifikationsanforderungen auch von den Verfassungsrichtern selbst als Element der Legitimation betrachtet. ${ }^{9}$ Die Auswahl der Richter und die Regelungen in Bezug auf ihre Amtszeit gelten auch aus politikwissenschaftlicher Sicht als entscheidende Variablen, wenn es um die Erklärung von Verfassungsgerichtsurteilen geht. ${ }^{10}$ In diesem Zusammenhang sind lange Amtszeiten, schwierige Abwahl und die Unmöglichkeit der Wiederwahl von besonderem Interesse. Sie konstituieren ein zentrales Element richterlicher Unabhängigkeit, ${ }^{11}$ indem sie politischen Druck von Richtern nehmen und ihnen erheblichen Handlungsfreiraum geben. Sie reduzieren damit aber auch die politische Verantwortlichkeit des Gerichts gegenüber den Bürgern.

9 Venice Commission: The Composition of Constitutional Courts, Science and Technique of Democracy, no. 20, 1997, 9 .

10 Hönnige, C.: Die Entscheidungen von Verfassungsgerichten - ein Spiegel ihrer Zusammensetzung?, in: Bräuninger, T./Behnke, J. (Hg.): Jahrbuch für Handlungs- und Entscheidungstheorie, Wiesbaden, 2006, 179-214.

11 Weber, A.: Generalbericht: Verfassungsgerichtsbarkeit in Westeuropa, in: Starck, C./Weber, A. (Hg.): Verfassungsgerichtsbarkeit in Westeuropa, Baden-Baden, 1986, 41-120, hier 54. 


\section{Politische Selektionskriterien}

Dem politischen Standort von Verfassungsrichtern wird häufig eine wichtige Rolle bei der Erklärung des Entscheidungsverhaltens beigemessen. Deshalb kommt der Auswahl von Verfassungsrichtern besondere Aufmerksamkeit zu. Bei der ersten Durchsicht der Besetzungsregeln in den einzelnen Ländern wird deutlich, dass diese insgesamt schwer zu klassifizieren sind, weshalb sich auch nur wenige entsprechende Versuche finden lassen. ${ }^{12}$

Um einen Überblick zu gewinnen, bietet sich zunächst an, die jeweilige Anzahl der Richter zu betrachten. Diese wird bereits meist in der Verfassung festgesetzt, spätestens jedoch im jeweiligen Verfassungsgerichtsgesetz. Die theoretischen Effekte der Gerichtsgröße sind umstritten: Einerseits nehmen mit steigender Richterzahl die Koordinations- und Verhandlungskosten zu, andererseits erlauben größere Gerichte ein stärkeres $\mathrm{Ma} ß$ an Spezialisierung und reduzieren die Fehlerwahrscheinlichkeit durch intensivere Diskussionen. ${ }^{13}$ Die Anzahl der Richter schwankt zwischen fünf (Estland) und 16 (Deutschland). Der am häufigsten vorkommende Besetzungstyp umfasst - entsprechend dem französischen Modell - neun Richter.

Die grundsätzliche Unterschiedlichkeit der Verfahren ermöglicht Regierung und Opposition auch unterschiedliche Beteiligungschancen bei der Besetzung der Richterposten. Hierbei sind insbesondere vier Fragen von Belang: (1) Welche Institutionen sind am Auswahlprozess beteiligt? (2) Läuft der Auswahlprozess zwischen den Institutionen sequentiell ab oder kann jede Institution, unabhängig von den anderen, einen Teil der Verfassungsrichter bestimmen? (3) Werden die Verfassungsrichter innerhalb der Institutionen gewählt oder nur ernannt? (4) Gilt im Fall einer Wahl eine einfache bzw. absolute Mehrheit als Entscheidungsquorum oder ist dazu eine Supermajorität erforderlich? Eine Übersicht über die Besetzungsverfahren gibt Tabelle $3^{14}$.

12 Venice Commission, a.a. O., 6-8; Ginsburg, T.: Judicial Review in New Democracies. Constitutional Courts in Asian Cases, Cambridge, 2003, 42-44.

13 Ginsburg, T., a.a. O., 47.

14 Eine detaillierte Übersicht über die zu Grunde liegenden Primärquellen, die analog zu den Tabellen strukturiert ist, findet sich bei Hönnige, C.: Verfassungsgericht, a. a. O., 273-276. 


\section{Beteiligte Institutionen}

Die Anzahl der am Auswahlprozess beteiligten Institutionen entscheidet zu einem erheblichen Maß über die Mitwirkungsmöglichkeiten von Regierung und Opposition in den parlamentarischen und semi-präsidentiellen Systemen der Europäischen Union. Je mehr Institutionen am Entscheidungsprozess beteiligt sind, desto eher wird die Opposition in der Lage sein, einen Teil der Verfassungsrichter zu bestimmen. Ein Beispiel dafür ist Österreich. Hier werden acht von 14 Richtern durch die Regierung ausgewählt, wobei der sie ernennende Bundespräsident an die Auswahl gebunden ist. Nationalrat und Bundesrat haben das Vorschlagsrecht für je drei weitere Richterposten und stimmen innerhalb der Institutionen mit einfacher Mehrheit ab. Sie legen dann dem Bundespräsidenten Dreierlisten vor, der aus diesen Listen wählen kann. ${ }^{15}$ Besitzt die Bundesregierung auch in der Zweiten Kammer die Mehrheit, ist die Opposition ohne Einfluss. Hat jedoch die Opposition im Bundesrat die Mehrheit, kann sie drei der 14 Verfassungsrichter bestimmen, während die Regierung und ihre Mehrheit im Nationalrat die übrigen elf Richter auswählen können.

Anzahl und Art der beteiligten Institutionen variieren im europäischen Vergleich erheblich. In allen Ländern ist die Legislative bei der Auswahl beteiligt und mit Ausnahme Italiens, Österreichs und Bulgariens wählt sie entweder den größten Teil der Richter aus bzw. stimmt über alle Richter ab. Handelt es sich um ein Zweikammersystem, ist außer in Polen die Zweite Kammer immer am Entscheidungsprozess beteiligt. Die interne Kandidatenfindung variiert ebenfalls. Sie kann entweder durch eine Gruppe Abgeordneter erfolgen (Polen), durch den Justizausschuss (in Deutschland der Bundesrat) oder durch einen proportional (in Deutschland der Bundestag) oder paritätisch (Ungarn) besetzen Richterwahlausschuss. In Polen kann zusätzlich das Parlamentspräsidium Vorschläge unterbreiten, in Litauen steht dieses Recht dem Parlamentspräsidenten zu. ${ }^{16}$

15 Korinek, K.: Die Verfassungsgerichtsbarkeit in Österreich, in: Starck, C./Weber, A. (Hg.): Verfassungsgerichtsbarkeit in Westeuropa, Baden-Baden, 1986, 149-178, hier $155 \mathrm{f}$.

16 Spuller, G.: Das Verfassungsgericht der Republik Ungarn, Frankfurt/M., 1998, 235; Mels, P.: Bundesverfassungsgericht und Conseil constitutionnel, München, 2003, 105; Steinsdorff, S. v.: Wer bestimmt die Verfassungsrichter? Auswahlverfahren in Deutschland, Frankreich und den USA zwischen politischem Kalkül und dem Ideal richterlicher Unabhängigkeit, Ms., 2002, 10; Brunner, G./Garlicki L.L.: Verfassungsgerichtsbarkeit in Polen. Analysen und Entscheidungssammlung 1986-1997, Baden-Baden, 1999, 25; Garlicki, L.L.: Das Verfassungsgericht im politischen Prozess, in: Luchterhandt, O. (Hg.): Neue Regierungssysteme in Osteuropa und der GUS, Berlin, 1996, 275-310, hier 287. 
Auch die Exekutive ist zumeist am Auswahlprozess beteiligt. Lediglich in Deutschland, Polen, Portugal und Ungarn hat die Regierung kein direktes Mitspracherecht, ist aber insofern indirekt eingebunden, als es sich um semi-präsidentielle bzw. parlamentarische Systeme handelt. Welcher Teil der Exekutive involviert ist, variiert. In Österreich, Spanien, Lettland und Litauen ist dies das Kabinett, ansonsten immer das Staatsoberhaupt. Der Staatspräsident kann entweder eine bestimmte Anzahl an Richtern autonom bestimmen (Frankreich, Italien, Bulgarien, Rumänien), dem Parlament zur Bestätigung vorschlagen (Estland, Slowakei, Tschechien) oder bekommt eine Liste, aus denen ein Kandidat ausgewählt werden muss (Österreich, Belgien, Slowakei).

Die Judikative wird in sieben der 17 Länder am Wahlprozess beteiligt. In Italien, Spanien und Bulgarien wählen die Obersten Gerichte selbst einen Teil der Richter, in Portugal kooptieren die zehn vom Parlament gewählten Richter drei weitere. In den baltischen Staaten besitzt das Gericht oder der Gerichtspräsident ein partielles Vorschlagsrecht, die Kandidaten müssen jedoch vom Parlament bestätigt werden.

\section{Sequenz oder Proporz}

Die Zusammenarbeit der beteiligten Institutionen lässt sich in zwei Typen unterscheiden: (1) sequentielle Verfahren, bei denen eine Institution ein Vorschlagsrecht besitzt und die andere Institution diesen annehmen oder ablehnen kann, und (2) proportionale Verfahren, bei denen die beteiligten Institutionen jeweils über eine bestimmte Anzahl von Richtern entscheiden können.

Ein Blick auf die Besetzungsverfahren zeigt, dass das proportionale Verfahren das bei weitem verbreitetste in Europa ist. Nur fünf der 17 Länder benutzen das sequentielle Verfahren: die drei baltischen Staaten Estland, Lettland und Litauen, sowie Slowenien und Tschechien. Die baltischen Staaten verteilen jedoch das Vorschlagsrecht auf die drei Gewalten, ${ }^{17}$ während die Slowakei und Tschechien dieses Privileg allein dem Staatspräsidenten zugestehen ${ }^{18}$ und es sich somit um eine Kopie des US-amerikanischen Modells handelt. In den proportionalen Systemen werden die Richter zu unterschiedlichen Anteilen auf Exekutive, Legislative und Judikative verteilt. Polen und Ungarn stellen das Ende des Spektrums

17 Schmidt, C., a. a. O., 474; Garlicki, L.L., a. a. O., 287.

18 Garlicki, L.L., a. a. O., 286. 
dar. Dort entscheidet nur eine Parlamentskammer über alle Richter, ${ }^{19}$ während in Spanien das Auswahlrecht auf Regierung, Deputiertenkongress, Senat und den Justizrat verteilt ist. ${ }^{20}$

Ginsburg $^{21}$ argumentiert, dass sequentielle Verfahren zu eher moderaten Kandidaten führen, wohingegen es bei institutionellem Proporz zu einer stärkeren Politisierung des Gerichts kommt, da es den Institutionen freisteht, ihre jeweiligen Agenten zu nominieren. ${ }^{22}$ Diese These ist allerdings etwas vereinfachend, geht sie doch von der impliziten Annahme eines divided government aus. Gehören Präsident und Senat zur gleichen Partei, kann auch dieses System parteipolitisch extrem positionierte Kandidaten erzeugen. Als entscheidender für den Einfluss der Opposition und die Fähigkeit einer Institution, parteipolitisch „extreme“ Kandidaten vorzuschlagen, erweist sich die Mehrheitsregel, wie im nächsten Abschnitt deutlich wird.

\section{Auswahlverfahren und Mehrheitsquorum}

Für die Opposition ist es von besonderem Interesse, nach welchem Wahlverfahren die Richter ausgesucht werden. Ist einer der Akteure berechtigt, einen Richter zu ernennen, ohne dafür einer Konsultations- oder Rechenschaftspflicht gegenüber anderen zu unterliegen? Oder werden die Verfassungsrichter gewählt und wenn ja, handelt es sich um eine einfache oder eine qualifizierte Mehrheit?

Die Venice Commission ${ }^{23}$ und Stone Sweet ${ }^{24}$ unterscheiden zwischen Systemen, die eine Ernennung durch einen oder mehrere autonome Akteure erlauben, die dafür niemandem Rechenschaft schuldig sind, Systemen, in denen eine Wahl stattfindet, und hybriden Systemen, in denen beide Verfahren zum Einsatz kommen. In der ersten Gruppe befindet sich nur Frankreich, wo der Staatspräsident, der Präsident der Nationalversammlung sowie der Präsident des Senates das Recht haben, je ein Drittel der Verfassungsrichter zu ernennen. ${ }^{25}$ Gemischte

19 Brunner, G./Garlicki, L.L., a.a.O., 25; Brunner, G./Sólyom, L.: Verfassungsgerichtsbarkeit in Ungarn. Analysen und Entscheidungssammlung 1990-1993, Baden-Baden, 1995, 20.

20 Llorente, F. R.: Die Verfassungsgerichtsbarkeit in Spanien, in: Starck, C./Weber, A. (Hg.): Verfassungsgerichtsbarkeit in Westeuropa, Baden-Baden, 1986, 243-278, hier 250.

21 Ginsburg, T., a. a. O., $43 \mathrm{f}$.

22 Ebd., 45.

23 Venice Commission, a.a. O., 6-9.

24 Stone Sweet, A.: Governing With Judges. Constitutional Politics in Europe, Oxford, 2000, 46.

25 Mels, P., a.a.O., 104. 
Systeme finden sich in Italien, Österreich, Spanien, Bulgarien und Rumänien. Alle anderen Länder benutzen das Wahlverfahren. Entgegen der landläufigen Meinung wird die Wahl überwiegend mit einfacher oder absoluter Mehrheit durchgeführt. Nur in sechs Ländern wird mit einer Supermajorität gewählt: Belgien, Deutschland, Italien und Ungarn (2/3-Mehrheit), Portugal (3/4-Mehrheit) sowie Spanien (3/5-Mehrheit). ${ }^{26}$

Tabelle 2: Klassifikation der Richterwahlverfahren

\begin{tabular}{|l|l|l|l|l|}
\hline Typ & Abfolge & Institutionen & Mehrheit & Länder \\
\hline 1 & Proporz & Legislative & einfache/absolute & Polen, Slowakei \\
\hline 2 & Proporz & Legislative & übergroße & $\begin{array}{l}\text { Deutschland, Belgien, } \\
\text { Ungarn }\end{array}$ \\
\hline 3 & Proporz & Mehrere Institutionen & einfache/absolute & $\begin{array}{l}\text { Österreich, Frankreich, } \\
\text { Rumänien, Bulgarien }\end{array}$ \\
\hline 4 & Proporz & Mehrere Institutionen & übergroße & $\begin{array}{l}\text { Spanien, Portugal, } \\
\text { Italien }\end{array}$ \\
\hline 5 & Sequenz & Exekutive/Legislative & einfache/absolute & $\begin{array}{l}\text { Tschechien, Slowe- } \\
\text { nien, Estland }\end{array}$ \\
\hline 6 & Sequenz & Exekutive/Legislative & übergroße & - \\
\hline 7 & Sequenz & Mehrere Institutionen & einfache/absolute & Lettland, Litauen \\
\hline 8 & Sequenz & Mehrere Institutionen & übergroße & - \\
\hline
\end{tabular}

Quelle: Eigene Darstellung

Welche Auswirkungen sind nun mit Blick auf den Einfluss von Regierung und Opposition zu erwarten? Das Mehrheitsquorum erlaubt der Opposition dann ein Mitspracherecht, wenn es sich um eine Supermajorität handelt, die die Regierung nicht alleine erreicht. Weder bei der Ernennung noch bei einfacher bzw. absoluter Mehrheit besteht die Möglichkeit dazu. Der analytische Unterschied zwischen Ernennung und der Wahl mit einfacher Mehrheit ist zwar nicht vernachlässigbar: Bei einer Ernennung kann der jeweils Ernennende diejenige Position auswählen, die seinem Idealpunkt am nächsten kommt, während bei einer Wahl der Kandidat die einfache Mehrheit der Abgeordneten erhalten muss. Betrachtet man aber den französischen Fall, stellt man fest, dass der Parlamentspräsident - der ein

26 Majoros, F.: Ungarische Verfassungsgerichtsbarkeit seit 1990, Köln, 1993, 17; Delpérée, F.: Die Verfassungsgerichtsbarkeit in Belgien, in: Starck, C./Weber, A. (Hg.): Verfassungsgerichtsbarkeit in Westeuropa, Baden-Baden, 1986, 343-362, hier 351; Benda, E.: Die Verfassungsgerichtsbarkeit der Bundesrepublik Deutschland, in: Starck, C./Weber, A. (Hg.): Verfassungsgerichtsbarkeit in Westeuropa, Baden-Baden, 1986, 121-148, hier 127; Luther, J.: Die italienische Verfassungsgerichtsbarkeit, BadenBaden, 1990, 64; Cardoso da Costa, J.M.M., a. a. O., 286; Llorente, F. R., a. a. O., 250. 
Drittel der Richter frei ernennen darf - von der Mehrheit des Parlamentes gewählt ist und deshalb seine ideologische Position nahe bei jener der Regierungsfraktionen liegen muss. Insofern kann man für eine dichotome Unterteilung die Ernennungen mit der Wahl durch einfache bzw. absolute Mehrheit eher zusammenfassen als mit der Wahl durch Supermajorität. Aus diesen drei Klassifikationsmerkmalen lassen sich acht Typen ableiten, die in Tabelle 2 abgebildet sind.

Tabelle 3: Richterwahlverfahren in 17 Ländern

\begin{tabular}{|c|c|c|c|c|}
\hline Staat & Anzahl & Wahlorgane & $\begin{array}{l}\text { (Interne) } \\
\text { Nominierung }\end{array}$ & Wahlquorum \\
\hline \multirow{2}{*}{ Belgien } & \multirow{2}{*}{12} & \multirow{2}{*}{$\begin{array}{l}\text { König wählt aus } \\
\text { Zweierliste aus }\end{array}$} & $\begin{array}{l}6 \text { Richter durch Abge- } \\
\text { ordnetenkammer }\end{array}$ & 2/3-Mehrheit \\
\hline & & & 6 Richter durch Senat & 2/3-Mehrheit \\
\hline \multirow{2}{*}{ Deutschland } & \multirow{2}{*}{16} & 8 durch Bundesrat & Justizminister & 2/3-Mehrheit \\
\hline & & 8 durch Bundestag & 12er Ausschuss & 2/3-Mehrheit \\
\hline \multirow{3}{*}{ Frankreich } & \multirow{3}{*}{9} & $\begin{array}{l}3 \text { durch Staatspräsi- } \\
\text { denten }\end{array}$ & $\begin{array}{l}\text { Keine Konsultations- } \\
\text { pflicht }\end{array}$ & Ernennung \\
\hline & & $\begin{array}{l}3 \text { durch Präsidenten } \\
\text { der Nationalver- } \\
\text { sammlung }\end{array}$ & $\begin{array}{l}\text { Keine Konsultations- } \\
\text { pflicht }\end{array}$ & Ernennung \\
\hline & & $\begin{array}{l}3 \text { Richter durch } \\
\text { Präsidenten des } \\
\text { Senats }\end{array}$ & $\begin{array}{l}\text { Keine Konsultations- } \\
\text { pflicht }\end{array}$ & Ernennung \\
\hline \multirow{3}{*}{ Italien } & \multirow{3}{*}{15} & $\begin{array}{l}5 \text { durch Staatspräsi- } \\
\text { denten }\end{array}$ & $\begin{array}{l}\text { Keine speziellen Rege- } \\
\text { lungen }\end{array}$ & Ernennung \\
\hline & & $\begin{array}{l}5 \text { durch beide Parla- } \\
\text { mentskammern }\end{array}$ & $\begin{array}{l}\text { Keine speziellen Rege- } \\
\text { lungen }\end{array}$ & 2/3-Mehrheit \\
\hline & & $\begin{array}{l}5 \text { durch Oberste } \\
\text { Gerichtshöfe }^{\mathrm{a}}\end{array}$ & $\begin{array}{l}\text { Keine speziellen Rege- } \\
\text { lungen }\end{array}$ & $\begin{array}{l}\text { Absolute } \\
\text { Mehrheit }\end{array}$ \\
\hline \multirow{3}{*}{ Österreich } & \multirow{3}{*}{$\begin{array}{c}14 \\
(+6)^{b}\end{array}$} & \multirow{3}{*}{$\begin{array}{l}\text { Formale Ernennung } \\
\text { durch Bundespräsi- } \\
\text { denten }\end{array}$} & $\begin{array}{l}8(+3) \text { durch Bundesre- } \\
\text { gierung }\end{array}$ & . \\
\hline & & & $3(+2)$ durch Nationalrat & $\begin{array}{l}\text { Einfache } \\
\text { Mehrheit }\end{array}$ \\
\hline & & & $3(+1)$ durch Bundesrat & $\begin{array}{l}\text { Einfache } \\
\text { Mehrheit }\end{array}$ \\
\hline \multirow{2}{*}{ Portugal } & \multirow{2}{*}{13} & 10 durch Parlament & $\begin{array}{l}\text { Vorschläge von mindes- } \\
\text { tens } 25 \text { Abgeordneten }\end{array}$ & 2/3-Mehrheit \\
\hline & & $\begin{array}{l}3 \text { durch Kooptation } \\
\text { des Gerichts selbst }\end{array}$ & $\begin{array}{l}\text { Ein geheimer Vorschlag } \\
\text { jedes Richters }\end{array}$ & $\begin{array}{l}\text { Absolute } \\
\text { Mehrheit }\end{array}$ \\
\hline
\end{tabular}

(Fortsetzung auf der nächsten Seite) 
(Fortsetzung)

\begin{tabular}{|c|c|c|c|c|}
\hline \multirow{4}{*}{ Spanien } & \multirow{4}{*}{12} & \multirow{4}{*}{$\begin{array}{l}\text { Formale Ernennung } \\
\text { durch König }\end{array}$} & 2 durch Regierung & - \\
\hline & & & 4 durch Kongress & 3/5-Mehrheit \\
\hline & & & 4 durch Senat & 3/5-Mehrheit \\
\hline & & & 2 durch Justizrat & $\begin{array}{l}\text { Einfache Mehr- } \\
\text { heit }\end{array}$ \\
\hline \multirow{3}{*}{ Bulgarien } & \multirow{3}{*}{12} & $\begin{array}{l}4 \text { durch Staatspräsi- } \\
\text { denten }\end{array}$ & $\begin{array}{l}\text { Keine speziellen Rege- } \\
\text { lungen }\end{array}$ & Ernennung \\
\hline & & 4 durch Parlament & $\begin{array}{l}\text { Keine speziellen Rege- } \\
\text { lungen }\end{array}$ & $\begin{array}{l}\text { Einfache Mehr- } \\
\text { heit }\end{array}$ \\
\hline & & $\begin{array}{l}4 \text { durch Oberste } \\
\text { Gerichte }^{\text {c }}\end{array}$ & $\begin{array}{l}\text { Keine speziellen Rege- } \\
\text { lungen }\end{array}$ & $\begin{array}{l}\text { Einfache Mehr- } \\
\text { heit }\end{array}$ \\
\hline Estland & $\begin{array}{c}9 \\
(19)^{\mathrm{d}}\end{array}$ & $\begin{array}{l}\text { Wahl durch Parla- } \\
\text { ment, Wahl durch } \\
\text { Gericht }\end{array}$ & $\begin{array}{l}\text { Vorschlag des Gerichts- } \\
\text { präsidenten durch } \\
\text { Staatspräsidenten; Vor- } \\
\text { schlag der Richter durch } \\
\text { Gerichtspräsidenten }\end{array}$ & $\begin{array}{l}\text { Einfache Mehr- } \\
\text { heit }\end{array}$ \\
\hline \multirow{3}{*}{ Lettland } & \multirow{3}{*}{7} & \multirow{3}{*}{$\begin{array}{l}\text { Wahl durch Parla- } \\
\text { ment }\end{array}$} & $\begin{array}{l}3 \text { durch mind. } 10 \text { Abge- } \\
\text { ordnete }\end{array}$ & \multirow{3}{*}{$\begin{array}{l}\text { Absolute Mehr- } \\
\text { heit }\end{array}$} \\
\hline & & & 2 durch Kabinett & \\
\hline & & & 2 durch Gericht selbst & \\
\hline \multirow{3}{*}{ Litauen } & \multirow{3}{*}{9} & \multirow{3}{*}{$\begin{array}{l}\text { Wahl durch Parla- } \\
\text { ment }\end{array}$} & $\begin{array}{l}3 \text { durch Staatspräsiden- } \\
\text { ten }\end{array}$ & \multirow{3}{*}{$\begin{array}{l}\text { Einfache Mehr- } \\
\text { heit }\end{array}$} \\
\hline & & & $\begin{array}{l}3 \text { durch Parlamentspräsi- } \\
\text { denten }\end{array}$ & \\
\hline & & & $\begin{array}{l}3 \text { durch Gerichtspräsi- } \\
\text { denten }\end{array}$ & \\
\hline Polen & 15 & $\begin{array}{l}\text { Wahl durch Parla- } \\
\text { ment (nur erste } \\
\text { Kammer) }\end{array}$ & $\begin{array}{l}\text { Mindestens } 50 \text { Abgeord- } \\
\text { nete oder Sejm-Präsi- } \\
\text { dium }\end{array}$ & $\begin{array}{l}\text { Absolute Mehr- } \\
\text { heit }\end{array}$ \\
\hline \multirow{3}{*}{ Rumänien } & \multirow{3}{*}{9} & $\begin{array}{l}3 \text { durch Staatspräsi- } \\
\text { denten }\end{array}$ & $\begin{array}{l}\text { Keine Konsultations- } \\
\text { pflicht }\end{array}$ & Ernennung \\
\hline & & $\begin{array}{l}3 \text { durch Abgeordne- } \\
\text { tenhaus }\end{array}$ & $\begin{array}{l}\text { Vorschlag durch } \\
\text { Rechtsausschuss }\end{array}$ & $\begin{array}{l}\text { Absolute Mehr- } \\
\text { heit }\end{array}$ \\
\hline & & 3 durch Senat & $\begin{array}{l}\text { Vorschlag durch Recht- } \\
\text { sausschuss }\end{array}$ & $\begin{array}{l}\text { Absolute Mehr- } \\
\text { heit }\end{array}$ \\
\hline Slowakei & $13^{\mathrm{e}}$ & $\begin{array}{l}\text { Staatspräsident wählt } \\
\text { aus Zweierliste }\end{array}$ & $\begin{array}{l}\text { Vorschlag durch Parla- } \\
\text { ment }\end{array}$ & $\begin{array}{l}\text { Einfache Mehr- } \\
\text { heit }\end{array}$ \\
\hline Slowenien & 9 & $\begin{array}{l}\text { Wahl durch Parla- } \\
\text { ment }\end{array}$ & Staatspräsident & $\begin{array}{l}\text { Absolute Mehr- } \\
\text { heit }\end{array}$ \\
\hline Tschechien & 15 & $\begin{array}{l}\text { Wahl durch Senat } \\
\text { oder Nichtreaktion }\end{array}$ & Staatspräsident & $\begin{array}{l}\text { Einfache Mehr- } \\
\text { heit }\end{array}$ \\
\hline Ungarn & $11^{\mathrm{f}}$ & $\begin{array}{l}\text { Wahl durch Parla- } \\
\text { ment }\end{array}$ & $\begin{array}{l}\text { Paritätischer Nominie- } \\
\text { rungsausschuss }\end{array}$ & 2/3-Mehrheit \\
\hline
\end{tabular}

Quelle: Eigene Darstellung 
Anmerkungen: a 3 durch Kassationsgericht, 1 durch Staatsrat, 1 durch Rechnungshof gewählt. ${ }^{\mathrm{b}}$ Ersatzmitglieder in Klammern angegeben. ${ }^{\mathrm{c}}$ Beteiligte Gerichte sind das oberste Kassationsgericht und das oberste Verwaltungsgericht. ${ }^{\mathrm{d}}$ Der Supreme Court hat 19 Mitglieder, von denen jedoch nur 9 in einer speziellen Kammer (Constiutional Review Chamber) für Normenkontrollverfahren zuständig sind. ${ }^{\mathrm{e}} \mathrm{Ab}$ 23.02. 2001 13, vorher 10 Richter. ${ }^{\mathrm{f}}$ Ursprünglich waren 15 Mitglieder geplant, die jedoch wegen Besetzungsstreitigkeiten nie erreicht wurden.

\section{Formale Qualifikationsanforderungen}

Die formalen Qualifikationsanforderungen lassen sich grob nach drei Punkten unterteilen: (1) Wird eine juristische Ausbildung vorausgesetzt und muss ein Teil der Verfassungsrichter zusätzlich eine besondere Qualifikation vorweisen? (2) Wird eine bestimmte Berufserfahrung verlangt oder diese indirekt durch ein Mindestalter sichergestellt? (3) Ist es erlaubt, gleichzeitig Mitglied einer Partei zu sein oder ist diese mit dem Richteramt inkompatibel? Die Übersicht über die entsprechenden Regelungen findet sich in Tabelle 4.

\section{a) Juristische Qualifikationen}

Alle Staaten mit Ausnahme Frankreichs verlangen von den Verfassungsrichtern ein abgeschlossenes Jurastudium, wobei die genauen Regelungen je spezifisch ausgestaltet sind. So ist in Österreich festgelegt, dass die Bundesregierung nur Juristen vorschlagen darf, die Verwaltungsbeamte, Rechtsprofessoren oder Richter sind, während Nationalrat und Bundesrat auch Anwälte vorschlagen dürfen. ${ }^{27}$ Auch in Ungarn müssen die Kandidaten entweder Rechtspraktiker mit Berufserfahrung oder „Theoretiker“, also Rechtsprofessoren, sein. ${ }^{28}$ In fast allen Ländern wird zusätzlich verlangt, dass die Richter über besonders herausgehobene Rechtskenntnisse verfügen müssen, wobei lediglich Belgien und Deutschland weitergehende juristische Kenntnisse festschreiben. In Deutschland müssen in jedem Senat jeweils 3/8 der Richter zuvor mindestens drei Jahre Richter an einem der obersten fünf Bundesgerichte tätig gewesen sein, ${ }^{29}$ während in Belgien je drei Richter aus jeder Sprachgruppe entweder für mindestens fünf Jahre am Kassationshof oder beim Staatsrat gewesen sein oder an einer Universität Rechtswissenschaft gelehrt haben müssen. ${ }^{30}$ In der Praxis handelt es sich bei Verfassungsgerichten oftmals um „Professorengerichte“ bzw. die Richter haben

27 Korinek, K., a.a.O., $155 \mathrm{f}$.

28 Spuller, G., a. a. O., 232.

29 Benda, E., a.a. O., 127.

30 Delpérée, F., a. a. O., $350 \mathrm{f}$. 
bereits Erfahrung im regulären Instanzenzug gesammelt. Selbst in Frankreich, wo es keinerlei Vorschriften hinsichtlich der beruflichen Qualifikation gibt, hat der überwiegende Anteil der Richter ein Jurastudium abgeschlossen.

\section{b) Mindestalter, Berufserfahrung und Altersgrenze}

Die meisten der 17 Verfassungsgerichte kennen entweder ein Mindestalter oder verlangen eine bestimmte Mindestberufserfahrung. Das Mindestalter ist dabei entweder auf 40 Jahre (Belgien, Deutschland, Frankreich, Slowakei, Slowenien, Tschechien) oder 45 Jahre (Ungarn) festgelegt. Lediglich in Frankreich entspricht es mit 18 Jahren dem normalen passiven Wahlrechtsalter. Eine vorgeschriebene Berufserfahrung erzeugt eine äquivalente Wirkung auf das mögliche Eintrittsalter der Richter. Geht man von einem Studienabschlussalter von etwa 25 Jahren aus, bedeutet dies, dass in Ländern, die 15 oder mehr Jahre Berufserfahrung verlangen, de facto ein Mindestalter von 40 Jahren erreicht wird (Italien, Spanien, Bulgarien, Rumänien, Slowakei, Ungarn) und dass auch bei zehn Jahren noch ein relativ hohes Mindesteintrittsalter von 35 entsteht (Österreich, Lettland, Litauen). Belgien stellt die untere Grenze mit fünf Jahren juristischer Berufserfahrung dar, wobei hier auch die Politiker acht Jahre Berufserfahrung aufweisen müssen. ${ }^{31}$ Lediglich Estland, Portugal und Polen kennen weder ein Mindestalter noch eine Mindestberufserfahrung.

Ob Mindestalter und Regelungen zur Ausbildung qualitativ hochwertige Urteile garantieren, ist umstritten. Epstein u. a. ${ }^{32}$ argumentieren für den US Supreme Court, dass sich in den Listen für ,große" Richter auch solche finden, welche die in europäischen Gerichten üblichen Mindeststandards nicht erfüllt hätten. Jedoch kann man tendenziell davon ausgehen, dass die Kriterien Mindestalter und Mindestberufserfahrung eine Reihe von möglicherweise nicht geeigneten Kandidaten fernhalten.

Komplementär zum Mindestalter wirkt die Altersgrenze für Verfassungsrichter. Epstein u.a. ${ }^{33}$ formulieren ihren Nutzen äußerst pietätvoll: ,Attempting to place outstanding nominees on the Bench is one thing; preserving the quality and distinction is quite another. "Angesichts der zumeist begrenzten Amtszeit erscheint der zusätzliche Nutzen von Altersgrenzen fraglich, weshalb sie auch in

31 Ebd., 351.

32 Epstein, L./Knight, J./Shvetsova, O.: Comparing Judicial Selection Systems, in: William and Mary Bill of Rights Journal, 10/1 (2001), 7-36, hier 11.

33 Ebd., 14. 
den meisten Fällen nicht vorkommen. Neben den Gerichten in Belgien, Österreich und Estland, die lebenslange Amtszeiten kennen, geben nur noch Deutschland, Lettland und Ungarn eine Grenze von 68 bis 70 Jahren vor, also knapp über dem üblichen Renteneintrittsalter. Die „Qualität“ erscheint somit bei den europäischen Gerichten durchgehend gewährleistet, zumindest in Bezug auf das Risiko alters- bzw. krankheitsbedingter Einschränkungen richterlicher Kompetenz.

\section{c) Parteimitgliedschaft}

Die Mitgliedschaft von Verfassungsrichtern in einer politischen Partei wird aus normativen Erwägungen oftmals kritisiert, da sie dem Ideal des unparteiischen Richters widerspricht. Der Besitz eines Parteibuchs erhöhe die Politisierung und reduziere die Eignung als Verfassungsrichter. Allerdings erlauben sechs der 17 Gerichte den Richtern die Mitgliedschaft in einer Partei (Belgien, Deutschland, Österreich, Italien, Spanien, Slowenien), bei zwei weiteren muss die Mitgliedschaft ruhen (Frankreich, Portugal), in allen anderen Gerichten ist die Mitgliedschaft nicht erlaubt. Hervorzuheben ist hier das belgische Schiedsgericht. Es ist das einzige Gericht, das explizit verlangt, dass die Hälfte der Richter sogar Politiker sein sollen. ${ }^{34}$ Die Inkompatibilität auf der anderen Seite betrifft vor allem die mittel- und osteuropäischen Gerichte, die mit der Ausnahme Sloweniens eine gleichzeitige Parteimitgliedschaft unterbinden. Die Gründe dafür finden sich im Transitionsprozess und dem Umgang mit den ehemaligen kommunistischen Eliten.

Beim Vergleich der institutionellen Regeln in Bezug auf die formale Qualifikation der Verfassungsrichter zeigt sich, dass diese innerhalb der 17 Länder weitestgehend ähnlich sind. Ausnahme ist Frankreich, das als einziges Land weder ein juristisches Studium noch ein Mindestalter oder eine gewisse Berufserfahrung verlangt. Nur drei Länder haben festgeschriebene Anforderungen, die über Jurastudium und Richteramt hinausgehen. Diesbezüglich ist die Varianz also sehr gering. Man kann Weber ${ }^{35}$ zustimmen, der für Westeuropa insgesamt feststellt, dass keine ernsthaften Zweifel an der Kompetenz der Verfassungsrichter bestehen können. 
Tabelle 4: Berufliche Qualifikationsanforderungen

\begin{tabular}{|c|c|c|c|c|c|c|c|}
\hline Staat & 离 & 胥 & 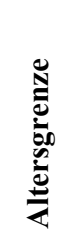 & 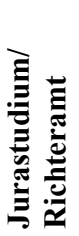 & $\begin{array}{l}\text { Besondere } \\
\text { juristische } \\
\text { Qualifika- } \\
\text { tionen }\end{array}$ & 包 & Anmerkungen \\
\hline Belgien & 40 & 5 & 70 & ja & $\begin{array}{l}\text { gehobene } \\
\text { juristische } \\
\text { Position }\end{array}$ & erlaubt & $\begin{array}{l}\text { Die Hälfte der Rich- } \\
\text { ter sollen Politiker } \\
\text { sein. Gleiche Anzahl } \\
\text { französisch- und } \\
\text { niederländischspra- } \\
\text { chiger Richter, davon } \\
\text { einer auch deutsch- } \\
\text { sprachig. }\end{array}$ \\
\hline Deutschland & 40 & - & 68 & ja & $\begin{array}{l}\text { pro Senat } 3 \\
\text { Richter aus } \\
\text { obersten } \\
\text { Gerichten }\end{array}$ & erlaubt & $\begin{array}{l}\text { Die Mitglieder der } \\
\text { obersten Gerichte } \\
\text { müssen mindestens } 3 \\
\text { Jahre dort tätig ge- } \\
\text { wesen sein. }\end{array}$ \\
\hline Frankreich & 18 & - & nein & nein & nein & $\begin{array}{c}\text { erlaubt } \\
\text { (ruhend) }\end{array}$ & - \\
\hline Italien & - & $20^{*}$ & nein & ja & nein & erlaubt & $\begin{array}{l}\text { * gilt nur für Anwäl- } \\
\text { te. }\end{array}$ \\
\hline Österreich & - & 10 & 70 & ja & nein & erlaubt & $\begin{array}{l}\text { Bundesregierung darf } \\
\text { nur Juristen vor- } \\
\text { schlagen, die Ver- } \\
\text { waltungsbeamte, } \\
\text { Rechtsprofessoren } \\
\text { oder Richter sind. }\end{array}$ \\
\hline Portugal & - & - & 70 & ja & nein & $\begin{array}{l}\text { erlaubt } \\
\text { (ruhend) }\end{array}$ & $\begin{array}{l}\text { Durch Parlament } \\
\text { gewählte Richter } \\
\text { müssen als Richter } \\
\text { ausgebildet sein. }\end{array}$ \\
\hline Spanien & - & 15 & nein & ja & nein & erlaubt & - \\
\hline Bulgarien & - & 15 & nein & ja & nein & $\begin{array}{l}\text { nicht } \\
\text { erlaubt }\end{array}$ & - \\
\hline Estland & - & - & 67 & ja & nein & $\begin{array}{l}\text { nicht } \\
\text { erlaubt }\end{array}$ & $\begin{array}{l}\text { Estnische Sprach- } \\
\text { kenntnisse sind } \\
\text { notwendig. }\end{array}$ \\
\hline Lettland & - & 10 & 70 & ja & nein & $\begin{array}{l}\text { nicht } \\
\text { erlaubt }\end{array}$ & - \\
\hline Litauen & - & 10 & nein & ja & nein & $\begin{array}{l}\text { nicht } \\
\text { erlaubt }\end{array}$ & - \\
\hline
\end{tabular}

(Fortsetzung auf der nächsten Seite) 
(Fortsetzung)

\begin{tabular}{|l|c|c|c|c|c|c|l|}
\hline Polen & - & - & nein & $\mathrm{ja}$ & nein & $\begin{array}{c}\text { nicht } \\
\text { erlaubt }\end{array}$ & $\begin{array}{l}\text { Besonders weitre- } \\
\text { chende Rechtskennt- } \\
\text { nisse erforderlich. }\end{array}$ \\
\hline Rumänien & - & 18 & nein & $\mathrm{ja}$ & nein & $\begin{array}{c}\text { nicht } \\
\text { erlaubt }\end{array}$ & $\begin{array}{l}\text { Ausgeprägte berufli- } \\
\text { che Ausgewiesenheit } \\
\text { wird verlangt. }\end{array}$ \\
\hline Slowakei & 40 & 15 & nein & $\mathrm{ja}$ & nein & $\begin{array}{c}\text { nicht } \\
\text { erlaubt }\end{array}$ & - \\
\hline Slowenien & 40 & - & nein & $\mathrm{ja}$ & nein & erlaubt & - \\
\hline Tschechien & 40 & - & nein & $\mathrm{ja}$ & nein & $\begin{array}{c}\text { nicht } \\
\text { erlaubt }\end{array}$ & - \\
\hline Ungarn & 45 & 20 & 70 & $\mathrm{ja}$ & $\begin{array}{c}\text { partiell } \\
\text { Professoren }\end{array}$ & $\begin{array}{c}\text { nicht } \\
\text { erlaubt }\end{array}$ & $\begin{array}{l}\text { Besondere Quote für } \\
\text { Hochschulprofesso- } \\
\text { ren. }\end{array}$ \\
\hline
\end{tabular}

Quelle: Eigene Darstellung

\section{Die Isolierung von politischem Druck}

Dieser Abschnitt betrachtet die institutionellen Regeln, mit denen Verfassungsrichter als individuelle Akteure oder das Gericht als kollektiver Akteur von politischem Druck isoliert werden können, indem ihnen die Anreize genommen werden, sich wegen drohendem Amtsentzug oder einer Überstimmung ihrer Urteile legislativen Mehrheiten zu beugen: (1) die Amtsdauer, (2) die Wiederwahl und (3) eine mögliche Abwahl, (4) ein reguläres Verfahren, wie Urteile überstimmt werden können, sowie (5) Verfassungsänderungen. Eine Übersicht über die verschiedenen Regeln findet sich in Tabelle 5.

\section{Amtsdauer}

Lebenslange Amtszeiten werden gemeinhin als Garant richterlicher Unabhängigkeit gesehen: Je länger die Amtszeit, desto unabhängiger sind die Richter von politischem Druck. ${ }^{36}$ Im Gegensatz zum US Supreme Court, den die überwiegende Mehrheit der Richter „mit den Füßen voraus“ verlässt, kennen europäische Verfassungsgerichte keine tatsächliche lebenslange Amtszeit. Lediglich Belgien, Österreich und Estland vermerken zwar die Amtszeit als lebenslang, geben aber gleichzeitig eine Altersgrenze an. Alle anderen europäischen Gerichte kennen nur beschränkte Amtszeiten von neun (Frankreich, Italien, Portugal, Spanien, 
Bulgarien, Litauen, Polen, Rumänien, Slowenien, Ungarn), zehn (Lettland, Tschechien) oder zwölf Jahren (Deutschland, Slowakei). Die Amtszeit von Verfassungsrichtern währt somit mindestens doppelt so lange wie eine normale Legislaturperiode, was eine gewisse Isolierung von politischem Druck bedeutet. Mit der begrenzten Amtszeit kann jedoch das Gericht graduell an neue politische Mehrheitsverhältnisse angepasst werden. ${ }^{37}$

\section{Wiederwahl und Abwahl}

Die Möglichkeit zur Wiederwahl kann durchaus die richterliche Unabhängigkeit gefährden. ${ }^{38}$ Ein Richter, der wiedergewählt werden möchte, könnte versucht sein, ein Urteil für seine wählende Institution annehmlich zu gestalten. Die Wiederwahl ist deshalb in den meisten Ländern nicht möglich. In Deutschland wurde sie bei der großen Gerichtsreform im Jahr 1970 abgeschafft, in Portugal im Jahr 1997. Ungarn kennt eine einmalige Wiederwahl ${ }^{39}$, und Tschechien ist das einzige Land, das die Wiederwahl generell erlaubt. ${ }^{40}$ In Estland werden die Richter des Supreme Court, die eigentlich lebenslange Amtszeiten kennen, für fünf Jahre in eine spezielle Verfassungskammer gewählt und dürfen einmal wiedergewählt werden. ${ }^{41}$

Eine weitere, ernsthafte Gefahr für die richterliche Unabhängigkeit stellt die Möglichkeit der Entlassung von Verfassungsrichtern dar, ${ }^{42}$ wenn entweder der Antrag oder die Entscheidung über die Entlassung außerhalb des Gerichts, insbesondere durch eine der wählenden Institutionen getroffen wird. In der überwiegenden Mehrheit der Fälle kann nur das Gericht selbst über die Entlassung eines Richters entscheiden (Österreich, Belgien, Bulgarien, Ungarn, Italien, Portugal, Rumänien, Spanien, Lettland, Litauen, Deutschland und Frankreich). In Estland (Justizkanzler und Parlament), Tschechien (Präsident), Slowenien (Parlament) und der Slowakei (Präsident) sind jedoch externe Akteure am Abwahlprozess beteiligt. $^{43}$

37 Garlicki, L.L., a. a. O., 288.

38 Venice Comission, a. a. O., 20; Epstein, L./Knight, J./Shvetsova, O., a. a. O., 22; Weber, A., a. a. O., 55.

39 Majoros, F., a. a. O., 17.

40 Venice Commission: Bulletin on Constitutional Case-Law. Special Edition Description of Courts, 1999, 40.

41 Schmidt, C., a. a. O., 473.

42 Epstein, L./Knight, J./Shvetsova, O., a.a.O., 22; Venice Commission: Composition of Constitutional Courts, a. a. O., 24-27; Weber, A., a. a. O., $56 \mathrm{f}$.

43 Venice Commission: Constitutional Case-Law, a. a. O., 41, 45, 79, 115, 112. 


\section{3. Überstimmung des Gerichts}

Die simpelste Form der Umgehung von Verfassungsgerichtsurteilen, die Richter möglicherweise zu strategischer Selbstbeschränkung animiert, sind formelle Überstimmungsverfahren. In Polen konnte der Sejm von 1986 bis 1999 mit einer Mehrheit von zwei Dritteln der anwesenden Mitglieder den Verfassungsgerichtshof ${ }^{44}$ überstimmen. ${ }^{45}$ In Rumänien war bis 2003 eine Überstimmung mit einer 2/3-Mehrheit in beiden Kammern möglich. Dort regelte Artikel 145 der Verfassung, dass nach der Annullierung eines Gesetzes durch das Verfassungsgericht dieses zu einer erneuten Beratung ins Parlament zurückgehen solle. Stimmte es erneut mit entsprechender Mehrheit für das Gesetz, galt dieses als endgültig verabschiedet. Auch der Staatspräsident - obwohl prinzipiell in der Lage, jedes Gesetz mit einem Veto zu verhindern - hatte dann keine Eingriffsmöglichkeit mehr. ${ }^{46}$ Portugal kennt die Überstimmung von Verfassungsgerichtsurteilen als einziges Land immer noch, allerdings nur bei der präventiven abstrakten Normenkontrolle. Dort kann das Urteil des Gerichts ebenfalls mit einer 2/3-Mehrheit im Parlament aufgehoben werden. ${ }^{47}$ Nur in Polen hat die Überstimmung eine effektive Rolle gespielt. Dort revidierte der Sejm im Zeitraum von 1986 bis 1999 elf Mal die Entscheidungen des Tribunal ${ }^{48}$ und damit etwa $10 \%$ aller Normannullierungen des Verfassungsgerichtshofes. ${ }^{49}$

\section{Verfassungsänderung}

Die dritte Methode, ein Urteil direkt zu umgehen, ist eine Verfassungsänderung. ${ }^{50}$ Damit wird dem Verfassungsgericht gleichsam die Entscheidungsgrund-

44 Dieses Verfahren ging auf das kommunistische Regime und den von ihm praktizierten sozialistischen Konstitutionalismus zurück. Mit der Schaffung eines Verfassungsgerichts wollte das Regime dem zunehmenden Protest im Land entgegenwirken, allerdings nicht ohne einen „Sicherheitsregler“ für den Fall einbauen, dass der Gerichtshof seine Selbstständigkeit bei der Verfassungsauslegung ,über Gebühr“ nutzen würde (Garlicki, L. L.: Das Verfassungsgericht und das Parlament. Die Zurückweisung von Entscheidungen des polnischen Verfassungsgerichtshofs durch den Sejm, in: Hofmann, M./Küpper, H. (Hg.): Kontinuität und Neubeginn. Staat und Recht in Europa zu Beginn des 21. Jahrhunderts, BadenBaden, 2001, 357-367, hier 357.)

45 Garlicki, L.L.: The Experience of the Polish Constitutional Court, in: Sadurski, W. (Hg.): Constitutional Justice, East and West, Den Haag, 2002, 265-282, hier 279.

46 Weber, R.: The Romanian Constitutional Court: In Search of Its Own Identity, in: Sadurski, W. (Hg.): Constitutional Justice, East and West, Den Haag, 2002, 283-308.

47 Magalhães, P. C., a. a. O., 111.

48 Garlicki, L. L.: The Experience of the Polish Constitutional Court, a. a. O., 279.

49 Ebd.

50 Stone Sweet, A., a. a. O. 
lage entzogen bzw. diese verändert. Allerdings sind die „Kosten“ für die Regierung sehr hoch, da die sehr häufig dafür benötigten qualifizierten Mehrheiten in der Regel nicht ohne Einbindung der Opposition zu erreichen sind. Je rigider die Verfassung ist, desto unwahrscheinlicher wird eine Reform und desto weniger werden Verfassungsrichter eine Verfassungsänderung als Bedrohung empfinden.

Tabelle 5: Isolation der Richter von politischem Druck

\begin{tabular}{|c|c|c|c|c|c|}
\hline Staat & Amtsdauer & Wiederwahl & 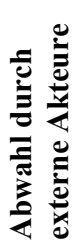 & 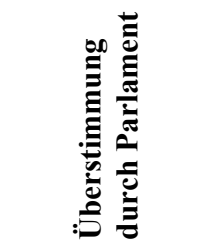 & 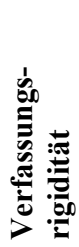 \\
\hline Belgien & Lebenszeit & nein & nein & nein & 9,5 \\
\hline Deutschland & 12 & nein (bis 1970: ja) & nein & nein & 6,0 \\
\hline Frankreich & 9 & nein & nein & nein & 4,0 \\
\hline Italien & 9 & nein & nein & nein & 4,0 \\
\hline Österreich & Lebenszeit & nein & nein & nein & 3,0 \\
\hline Portugal & 9 & nein (bis 1997: ja) & nein & 2/3 Mehrheit $^{\mathrm{a}}$ & 3,0 \\
\hline Spanien & 9 & nein & nein & nein & 6,0 \\
\hline Bulgarien & 9 & nein & nein & nein & 3,0 \\
\hline Estland & Lebenszeit $(5)^{b}$ & $\mathrm{ja}^{\mathrm{b}}$ & ja & nein & 2,0 \\
\hline Lettland & 10 & nein & nein & nein & 4,0 \\
\hline Litauen & 9 & nein & nein & nein & 4,0 \\
\hline Polen & 9 & nein & nein & $\begin{array}{c}\text { nein (bis 1999: } \\
\text { 2/3 Mehrheit) }\end{array}$ & 3,0 \\
\hline Rumänien & 9 & nein & nein & $\begin{array}{c}\text { nein }^{c} \text { (bis 2003: } \\
\text { 2/3-Mehrheit) }\end{array}$ & 8,0 \\
\hline Slowakei & 12 & nein & ja & nein & 3,0 \\
\hline Slowenien & 9 & nein & ja & nein & 3,0 \\
\hline Tschechien & 10 & ja & ja & nein & 4,0 \\
\hline Ungarn & 9 & einmal & nein & nein & 3,0 \\
\hline
\end{tabular}

Quelle: Eigene Darstellung

Anmerkungen: Verfassungsrigidität nach Lorenz, A.: How to Measure Constitutional Rigidity - Four Concepts and Two Alternatives, in: Journal of Theoretical Politics, 17/3 (2005), 339-361. ${ }^{a}$ Die Überstimmung ist nur bei einer präventiven Normenkontrolle möglich. ${ }^{b}$ Die Richter werden aus der Mitte des Gerichts, dem sie lebenslang angehören, bei einmaliger Wiederwahlmöglichkeit für 5 Jahre in die Verfassungskammer gewählt. ${ }^{\mathrm{c}}$ Die Überstimmung ist bei Referendumskontrolle weiterhin möglich. 
Um die Rigidität von Verfassungen zu messen, wird auf den Index von Lorenz ${ }^{51}$ zurückgegriffen. Dieser berücksichtigt systematisch die Anzahl der an der Verfassungsänderung beteiligten Kammern, die Mehrheitsregeln sowie zusätzliche Anforderungen wie eine Zustimmung des Staatspräsidenten oder ein Verfassungsreferendum, wobei auch hier explizit die Mehrheitsanforderungen berücksichtigt werden. Die fehlenden Werte für Estland, Lettland, Rumänien und die Slowakei wurden entsprechend den Kodierregeln hinzugefügt (Tabelle 5).

\section{Zugangswege zum Verfassungsgericht}

Wichtiger Schlüssel und notwendige Bedingung für die Nutzung des Verfassungsgerichts und damit dessen Einfluss ist der Zugang zum Gericht. ${ }^{52}$ Dabei sind zwei Fragen von Bedeutung: (1) Welche Verfahrensarten und Kompetenzen kennen Verfassungsgerichte, insbesondere solche, die es ihnen erlauben, eine Norm im Einzelfall oder generell zu beanstanden? (2) Welche Akteure haben das Recht, das Gericht anzurufen, und an welche Bedingungen (Klagequoren etc.) ist dies geknüpft? Die Klassifikation der Kompetenzen von Verfassungsgerichten wird in der Literatur unterschiedlich vorgenommen. ${ }^{53}$ Der vorliegende Beitrag folgt hier im Wesentlichen der für Westeuropa entwickelten Einteilung von $\mathrm{We}$ ber, da die Verfahrensarten der osteuropäischen Gerichte nicht stark davon abweichen. ${ }^{54}$ Die Gliederung erfolgt nach Normenkontrollen (präventiv abstrakt, repressiv abstrakt und konkret), Kompetenzkonflikten (horizontal, vertikal), Verfassungsbeschwerden sowie Wahl- und Mandatsprüfung einschließlich Referendumskontrolle. Verfassungsschutzverfahren (Parteienverbot, Grundrechtsverwirkung, Minister- und Präsidentenanklagen) werden nicht explizit diskutiert (Tabelle 6).

\section{Abstrakte Normenkontrolle}

Bei einer Normenkontrolle wird die Vereinbarkeit eines Gesetzes oder einer Verordnung mit der Verfassung überprüft. Normenkontrollen stellen somit den

51 Lorenz, A., a. a. O.

52 Ginsburg, T., a.a.O., 36.

53 Vgl. Brünneck, A.v.: Verfassungsgerichtsbarkeit in den westlichen Demokratien, Baden-Baden, 1992, 36-48; Venice Commission: Models of Constitutional Jurisdiction, no. 2, 1993; Weber, A., a. a. O., 62101.

54 Garlicki, L. L.: Das Verfassungsgericht im politischen Prozess, a. a. O., 292. 
Kernbestand der Kompetenzen von Verfassungsgerichten dar. ${ }^{55}$ Bei der abstrakten Normenkontrolle werden Gesetze und Verordnungen losgelöst vom Einzelfall geprüft und mithin einer objektiven Verfassungskontrolle unterzogen, egal ob es sich um die Verfassung des Zentralstaates, die eines Gliedstaates oder um die Kompetenzverteilung handelt. Nicht geprüft wird jedoch der Schutz konkreter Individualrechte. ${ }^{56}$ Der Zeitpunkt des Verfahrens kann entweder vor der Verkündung eines Gesetzes sein (präventive abstrakte Normenkontrolle) oder nach der Verkündung (repressive Kontrolle). ${ }^{57}$

Die präventive abstrakte Normenkontrolle findet sich in einigen Ländern explizit in Bezug auf völkerrechtliche Verträge und verfassungsergänzende Gesetze, kommt aber bei einfachen Gesetzen äußerst selten vor. Lediglich Frankreich und Rumänien sowie in eingeschränkter Form Estland, Portugal, Polen und Ungarn kennen die präventive abstrakte Normenkontrolle bei normalen Gesetzen.

Tabelle 6: Verfassungsgerichtliche Verfahrensarten

\begin{tabular}{|l|c|c|c|c|c|c|}
\hline \multirow{2}{*}{ Staat } & \multicolumn{3}{|c|}{ Normenkontrolle } & \multicolumn{2}{c|}{ Kompetenzstreit } & \multirow{2}{*}{$\begin{array}{c}\text { Verfassungs- } \\
\text { beschwerde }\end{array}$} \\
\cline { 2 - 5 } & \multicolumn{2}{|c|}{ abstrakt } & \multirow{2}{*}{ konkret } & horizontal & vertikal & \\
\cline { 2 - 5 } & präventiv & repressiv & & & nein \\
\hline Belgien & nein & ja & ja & ja & ja & ja \\
\hline Deutschland & nein & ja & ja & ja & ja & nein \\
\hline Frankreich & ja & nein & nein & ja & nein & nein \\
\hline Italien & nein & ja & ja & ja & ja & ja \\
\hline Österreich & nein & ja & ja & ja & ja & nein \\
\hline Portugal & ja & ja & ja & nein & ja & ja \\
\hline Spanien & nein & ja & ja & ja & ja & nein \\
\hline Bulgarien & nein & ja & ja & ja & nein & nein \\
\hline Estland & ja & ja & ja & nein & nein & ja \\
\hline Lettland & nein & ja & ja & ja & nein & nein \\
\hline Litauen & nein & ja & ja & nein & nein & ja \\
\hline Polen & ja & ja & ja & ja & nein & nein \\
\hline Rumänien & ja & nein & ja & nein & nein & ja \\
\hline Slowakei & nein & ja & ja & ja & nein & ja \\
\hline Slowenien & nein & ja & ja & ja & nein & ja \\
\hline Tschechien & nein & ja & ja & ja & nein & ja \\
\hline Ungarn & ja & ja & ja & ja & nein & \\
\hline
\end{tabular}

Quelle: Eigene Darstellung

55 Ebd., 289.

56 Weber, A., a. a. O., 68.

57 Synonym verwendet werden die Begriffe a priori für die präventive und a posteriori für die repressive abstrakte Normenkontrolle. 
Die repressive abstrakte Normenkontrolle ist ungleich weiter verbreitet und neben der konkreten Normenkontrolle das am häufigsten vorzufindende Verfahren. Lediglich Frankreich und das institutionell daran angelehnte rumänische Gericht kennen es überhaupt nicht. ${ }^{58}$ In diesen beiden Ländern ersetzt das präventive

Tabelle 7: Antragsteller und Klagequoren bei der abstrakten Normenkontrolle

\begin{tabular}{|c|c|c|c|c|c|c|c|c|}
\hline \multirow[b]{2}{*}{ Staat } & \multicolumn{2}{|c|}{ Exekutive } & \multicolumn{2}{|c|}{ Erste Kammer } & \multicolumn{2}{|c|}{ Zweite Kammer } & \multicolumn{2}{|c|}{$\begin{array}{c}\text { Gliedstaat/ } \\
\text { Autonome } \\
\text { Region }\end{array}$} \\
\hline & 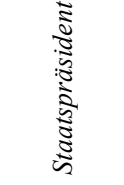 & 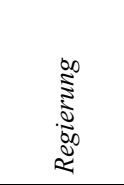 & 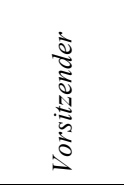 & 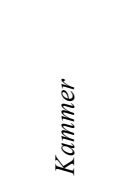 & 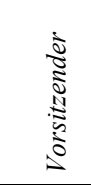 & 离 & 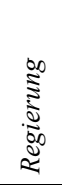 & 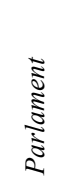 \\
\hline Belgien & nein & Regierung & \multicolumn{2}{|c|}{ Vorsitzender und $2 / 3^{a}$} & \multicolumn{2}{|c|}{$\begin{array}{c}\text { Vorsitzender und } \\
2 / 3^{\mathrm{a}}\end{array}$} & ja & ja \\
\hline Deutschland & nein & nein & nein & $1 / 3$ & nein & nein & ja & nein \\
\hline Frankreich $^{\mathrm{b}}$ & $\mathrm{ja}$ & Premier & $\mathrm{ja}$ & 60 & $\mathrm{ja}$ & 60 & n.a. & n.a. \\
\hline Italien & nein & nein & nein & nein & nein & nein & ja & nein \\
\hline Österreich & nein & nein & nein & $1 / 3$ & nein & $1 / 3$ & ja & nein $^{\mathrm{c}}$ \\
\hline Portugal & ja & Premier & $\begin{array}{c}\text { repressiv: } \\
\text { nein } \\
\text { präventiv: } \\
\text { ja }\end{array}$ & $\begin{array}{c}\text { repressiv: } \\
1 / 10 \\
\text { präventiv: } \\
1 / 5\end{array}$ & n.a. & n.a. & ja & ja \\
\hline Spanien & nein & Premier & nein & 50 & nein & 50 & $\mathrm{ja}$ & ja \\
\hline Bulgarien & $\mathrm{ja}$ & Regierung & nein & $1 / 5$ & n.a. & n.a. & n.a. & n.a. \\
\hline Estland & $\mathrm{ja}^{\mathrm{d}}$ & nein & nein & nein & n.a. & n.a. & n.a. & n.a. \\
\hline Lettland & ja & Regierung & nein & 20 & n.a. & n.a. & n.a. & n.a. \\
\hline Litauen & nein & Regierung & nein & $1 / 5$ & n.a. & n.a. & n.a. & n.a. \\
\hline Polen & ja & Premier & ja & 50 & $\mathrm{ja}$ & 30 & n.a. & n.a. \\
\hline Rumänien $^{\text {b }}$ & ja & Regierung & ja & 50 & ja & 25 & n.a. & n.a. \\
\hline Slowakei & ja & Regierung & nein & $1 / 5$ & n.a. & n.a. & n.a. & n.a. \\
\hline Slowenien & nein & Regierung & nein & $1 / 3$ & nein & ja & n.a. & n.a. ${ }^{\mathrm{e}}$ \\
\hline Tschechien & ja & nein & nein & 41 & n.a. & 17 & n.a. & n.a. \\
\hline \multirow[t]{2}{*}{ Ungarn } & $\begin{array}{c}\text { präventiv: } \\
\text { ja }\end{array}$ & $\begin{array}{l}\text { präventiv: } \\
\text { nein }\end{array}$ & $\begin{array}{l}\text { präventiv: } \\
\text { nein }\end{array}$ & $\begin{array}{l}\text { präventiv: } \\
50\end{array}$ & n.a. & n.a. & n.a. & n.a. \\
\hline & \multicolumn{8}{|c|}{$\begin{array}{l}\text { repressiv: jedermann } \\
\end{array}$} \\
\hline
\end{tabular}

Quelle: Eigene Darstellung

Anmerkungen: ${ }^{a}$ Das Antragsrecht steht den Vorsitzenden aller Kammern nach einer Beauftragung durch ein Votum mit 2/3 Mehrheit der Abgeordenten zu. ${ }^{b}$ Nur präventive, keine repressive abstrakte Normenkontrolle. ${ }^{\mathrm{c}}$ Abweichende Regelungen sind per Landesverfassungsgesetz möglich, so dass den Landtagen auch eine Antragsbefugnis durch 1/3 der Mitglieder zusteht. ${ }^{\mathrm{d}}$ Antragsrecht des Präsidenten beschränkt auf die präventive abstrakte Normenkontrolle. ${ }^{\mathrm{e}} \mathrm{Ab}$ 20.06. $2006 \mathrm{ja}$, vorher n.a.

58 Garlicki, L. L.: Das Verfassungsgericht im politischen Prozess, a. a. O., 294. 
Verfahren das repressive. Die Antragsbefugnis für abstrakte Normenkontrollen für einfachgesetzliche Regelungen ist im europäischen Vergleich sehr heterogen. $\mathrm{Zu}$ den Antragstellern gehören Akteure der Exekutive, insbesondere der Staatspräsident, das Kabinett oder der Regierungschef, die erste Parlamentskammer, sofern vorhanden die zweite Parlamentskammer, Regionalregierungen, Regionalparlamente und eine ganze Reihe weiterer Institutionen. Eine Übersicht über Antragsteller und Klagequoren findet sich in Tabelle 7.

\section{Konkrete Normenkontrolle}

Bei einer konkreten Normenkontrolle wird eine Norm anlässlich eines konkreten Falles, d.h. ihrer Anwendung bei einem Rechtsstreit, auf Verfassungskonformität überprüft. ${ }^{59}$ Variationen entstehen bei der Frage, ob die regulären Gerichte eine Vorlagepflicht oder ein Vorlagerecht haben und ob alle Gerichte im Instanzenzug einen Fall dem Verfassungsgericht zur Entscheidung zuweisen können.

Vorlagepflicht herrscht in allen Ländern, die dem konzentrierten Modell der Verfassungsgerichtsbarkeit folgen, also nicht Portugal ${ }^{60}$ und Estland $^{61}$. In Bulgarien beispielsweise ist die Antragsbefugnis auf die obersten Gerichte beschränkt, ${ }^{62}$ während in Polen sämtliche Gerichte im Instanzenzug seit 1997 vorlagepflichtig sind, wenn sie eine Kollision vermuten. Alle Länder mit Ausnahme Frankreichs kennen die konkrete Normenkontrolle.

\section{Horizontale und vertikale Kompetenzstreitigkeiten}

In diese Gruppe fallen föderale und quasi-föderale Streitigkeiten von Zentralstaat, Gliedstaaten und autonomen Gemeinschaften bzw. Regionen ${ }^{63}$ (vertikale Konflikte) sowie Streitigkeiten zwischen Staatsorganen auf Zentralstaatsebene (horizontale Konflikte), wobei erstere schwierig von abstrakten Normenkontrollen abzugrenzen sind, bei denen Gliedstaaten oder Regionen Antragsrechte besitzen. $^{64}$ Vertikale Kompetenzstreitigkeiten finden sich in Belgien, Deutschland,

59 Weber, A., a. a. O., 74.

60 Cardoso da Costa, J.M.M., a. a. O., 291.

61 Schmidt, C., a. a. O., 474.

62 Nanova, M.: Verfassungsgerichtsbarkeit in Bulgarien, Baden-Baden, 2002, 105.

63 Streitigkeiten von Gliedstaaten bzw. Regionen untereinander, wie sie häufiger in Italien, Belgien oder Spanien vorkommen (Weber, A., a. a. O., 82), werden hier nicht behandelt. Konflikte zwischen Zentralstaat und Kommunen werden ebenfalls nicht erörtert.

64 Weber, A., a. a. O., 67. 
Italien, Österreich, Spanien und Portugal. Allseitige oder partielle horizontale Kompetenzkonflikte, bei denen die Rechte und Pflichten oberster Staatsorgane in einem schiedsrichterlichen Verfahren geregelt werden, gibt es in fast allen Ländern: Belgien, Deutschland, Frankreich, Italien, Österreich, Spanien, Bulgarien, Lettland, Polen, Slowakei, Slowenien, Tschechien und Ungarn. ${ }^{65}$

\section{Verfassungsbeschwerde}

Verfassungsbeschwerden dienen dazu, die Grundrechte des Einzelnen gegen Akte von Exekutive, Legislative und Judikative zu schützen. Die Klage kann sich dabei entweder gegen den Akt selbst (echte Verfassungsbeschwerde) oder gegen die dahinter stehende Norm (unechte Beschwerde) richten. In manchen Ländern ist die Reichweite der Beschwerde auf Akte der Exekutive (z.B. in Österreich) oder Akte der Exekutive und Judikative (z. B. in Spanien) begrenzt, was aber teilweise durch Individualnormenkontrollen kompensiert wird (so in Österreich und Spanien). Charakteristisches Merkmal dieses Verfahrentyps ist, dass der Kläger, um es mit der Prüfungsformel des Bundesverfassungsgerichts zu sagen, ,selbst, gegenwärtig und unmittelbar“ in solchen Rechten verletzt sein muss ${ }^{66}$ Dieses Verfahren besteht in unterschiedlichen Ausprägungen in Deutschland, Österreich, Spanien, Lettland, Polen, Slowakei, Slowenien, Tschechien und Ungarn.

\section{Sonstige Kompetenzen}

Darüber hinaus finden sich noch atypische Verfahrensarten im Zuständigkeitskatalog der Verfassungsgerichte. Die allgemein verbindliche Gesetzesauslegung (Polen) und die Verfassungsauslegung (Ungarn, Slowakei) gehören dazu. Mit diesen Verfahren kann die Verfassung bzw. können einfache Gesetze verbindlich interpretiert werden, ohne dass ein konkreter Disput geführt wird. ${ }^{67}$

Parteienverbot und Grundrechtsverwirkung gehören - wie die hier nicht näher behandelten Verfahren der Ministeranklage und der Sicherung der Funktionsfähigkeit der Verfassungsorgane (z. B. Präsidentenanklage, Amtsunfähigkeit des

65 Weber, A., a. a.O., 79-85; Stern, K.: Neue Entwicklungen der Verfassungsgerichtsbarkeit in den Staaten Ostmitteleuropas, in: Hofmann, M./Küpper, H. (Hg.): Kontinuität und Neubeginn. Staat und Recht in Europa zu Beginn des 21. Jahrhunderts, Baden-Baden, 2001, 150-164, hier 159; Garlicki, L.L.: Das Verfassungsgericht im politischen Prozess, a. a. O., 289.

66 Schlaich, K./Korioth, S.: Das Bundesverfassungsgericht, München, 2001, 149.

67 Stern, K., a.a. O., 160. 
Staatschefs) - zu den Verfassungsschutzverfahren. ${ }^{68}$ Wahl- und Mandatsprüfungen sind weitere gängige Zuständigkeiten eines Verfassungsgerichts. Zu ihnen zählen sowohl die Prüfung von Parlamentswahlen als auch der Wahl zum Staatsoberhaupt bzw. der Verfassungsmäßigkeit von Referenden. ${ }^{69}$

Verfassungsgerichte verfügen im Allgemeinen über ein breites Spektrum an Kompetenzen, sind aber sehr unterschiedlich damit ausgestattet. Während die nach dem Vorbild Deutschlands und Österreichs geformten mitteleuropäischen Gerichte sowie Portugal und Spanien über eine Vollausstattung verfügen, sind jene der baltischen Staaten in ihrem Aktionsradius deutlich eingeschränkter.

\section{Die Organisation von Verfassungsgerichten}

Arenen, zentrale Akteure und Entscheidungsregeln bilden das intra-institutionelle strategische Umfeld der Richter. Diese strukturieren zu einem erheblichen Maße, inwieweit einzelne Richter in der Lage sind, ihre Positionen durchzusetzen und welche Entscheidungsergebnisse möglich sind (Tabelle 8).

\section{Arenen: Plenum, Senate und Kammern}

Grundsätzlich lassen sich Ein- und Zweikammersysteme in Form von Senaten sowie eine Aufteilung in Kammern bei der Gerichtsorganisation unterscheiden. Von analytischem Interesse ist die Unterteilung in Senate und Kammern vor allem dann, wenn ihre politische Besetzung von der des Gesamtgerichts abweicht. So lässt sich beispielsweise in den frühen Jahren der Bundesrepublik ein „roter“ und ein „schwarzer“ Senat identifizieren. ${ }^{70}$

Das Einkammergericht, bei dem die Richter im Plenum tagen, kommt am häufigsten vor. Nur in Deutschland ${ }^{71}$ sowie in den institutionell daran orientierten Gerichten in Portugal ${ }^{72}$ und Spanien ${ }^{73}$ findet sich eine Mehrkammernlösung in Form von ständigen Senaten. Diese ist in Deutschland durch eine gesetzliche Zuständigkeitsregelung für die Senate am deutlichsten ausgeprägt. In Portugal

68 Weber, A., a. a. O., 96.

69 Ebd., $94 \mathrm{f}$.

70 Wesel, U.: Der Gang nach Karlsruhe. Das Bundesverfassungsgericht in der Geschichte der Bundesrepublik, München, 2004, 97-105.

71 Benda, E., a. a. O., 126.

72 Cardoso da Costa, J.M.M., a. a. O., 296.

73 Llorente, F. R., a. a. O., 252. 
dagegen sind die Senate nicht spezialisiert und in Spanien in ihrer Zuständigkeit begrenzt. $^{74}$

Oftmals findet sich eine Unterteilung nach Richterausschüssen, auch Kammern genannt, so in Belgien, Deutschland, Österreich, Portugal, Spanien, Lettland, Polen, der Slowakei, Slowenien, Tschechien und Ungarn. Diese Richterausschüsse dienen üblicherweise dazu, die Arbeitslast des Gesamtgerichts zu reduzieren, indem ihnen entweder die Zulässigkeitsprüfungen, die komplette Erledigung von Verfassungsbeschwerden oder konkreten Normenkontrollen anvertraut werden. Abstrakte Normenkontrollen unterliegen jedoch immer dem Plenumsvorbehalt bzw. der Entscheidung der Senate in Deutschland.

\section{Zentrale Akteure: Berichterstatter und Gerichtspräsident}

Zwei zentrale Akteure lassen sich im Entscheidungsprozess des Gerichts identifizieren: den Berichterstatter, auch Rapporteur genannt, und den Gerichtspräsidenten bzw. der den Vorsitz führende Richter. Die Aufgabe des Berichterstatters ist es, das Urteil zu entwerfen, über das dann im Plenum bzw. im jeweiligen Senat abgestimmt wird. Damit kommen dem Berichterstatter erhebliche Möglichkeiten zur Agendasetzung innerhalb des Gerichts zu. ${ }^{75}$ Es lassen sich vier Verfahren identifizieren, nach denen Berichterstatter ausgewählt werden: Sie werden durch das Los (Portugal, Slowakei) oder in alphabetischer Reihenfolge (Slowenien) bestimmt, durch das Plenum des Gerichts gewählt (Deutschland, Spanien, Estland) oder durch den Gerichtspräsidenten ernannt. Aus Sicht von Regierung und Opposition besteht somit eine erhebliche Unsicherheit darüber, wer in einem Fall die einflussreiche Position des Berichterstatters übernimmt.

Dem Präsidenten des Gerichts bzw. dem Vizepräsidenten kommt eine ganze Reihe unterschiedlicher Funktionen zu. Sie haben Aufsichtspflicht gegenüber dem Personal, sie repräsentieren das Gericht nach außen und übernehmen Führungsfunktionen. ${ }^{76}$ Aus theoretischer Sicht ist von besonderem Interesse, ob sie den Berichterstatter benennen dürfen und im Falle eines Patts bei der Abstimmung das ausschlaggebende Votum haben. Dann verfügen sie über die Möglich-

74 Weber, A., a. a. O., 59.

75 In Portugal verliert ein Berichterstatter den Fall, wenn er mit dem Urteil oder der Begründung nicht zumindest die Hälfte der Richter für sich gewinnen kann. Der Fall wird dann einem anderen Berichterstatter übertragen (Venice Commission: Bulletin on Constitutional Case-Law, a. a. O., 101.)

76 Weber, A., a.a. O., $59 \mathrm{f}$. 
keit, sowohl die Agenda als auch die Abstimmung zu einem erheblichen Teil zu kontrollieren.

Hinsichtlich der Amtszeit der Gerichtspräsidenten lassen sich zwei Grundmodelle erkennen: Entweder sie werden für eine begrenzte Amtszeit gewählt (viereinhalb Jahre: Portugal; drei Jahre: Italien, Spanien, Bulgarien, Lettland, Rumänien, Slowenien, Ungarn; zwei Jahre: Portugal; ein Jahr: Belgien), wobei dann eine Wiederwahl zulässig ist, oder sie werden für ihre komplette Amtsdauer als Richter von einem Wahlorgan ernannt (Deutschland, Frankreich, Österreich, Estland, Litauen, Polen, Slowakei, Tschechien).

Die Auswahl erfolgt entweder durch das Gericht selbst oder durch eine der Wahlinstitutionen, wobei zumeist der Exekutive und insbesondere dem Staatspräsidenten dieses Recht zukommt. Lediglich in Deutschland wählen Bundestag und Bundesrat den Gerichtspräsidenten im Wechsel. ${ }^{77}$ Zwischen Wahlverfahren und Amtszeit der Gerichtspräsidenten lässt sich insofern ein Zusammenhang finden, als dass in jenen Ländern, in denen die Gerichtspräsidenten vom Gericht selbst gewählt werden, die Amtszeit begrenzt ist, während bei der Auswahl des Gerichtspräsidenten durch ein Wahlorgan die Amtszeit des Gerichtspräsidenten an die gesamte Amtsdauer als Richter gekoppelt ist. Im letzten Fall erscheint das Entscheidungsverhalten des Gerichts für Regierung und Opposition mit weniger Unsicherheit behaftet als im Falle der begrenzten Amtszeit und bei Wahl durch das Gericht.

\section{Entscheidungsregeln}

Von offensichtlicher Bedeutung sind die Abstimmungsregeln innerhalb des Gerichts. Je höher das Abstimmungsquorum, desto schwieriger wird eine Einigung und desto eher wird ein Gesetz nicht für verfassungswidrig erklärt. Ceteris paribus reduziert sich der Raum zur Einigung und der Aktionsradius des Agendasetzers bei steigenden Mehrheitserfordernissen. ${ }^{78}$

Als Mehrheitsregeln bei Normenkontrollverfahren werden überwiegend die einfache Mehrheit (Deutschland, Frankreich, Italien, Spanien, Portugal, Estland, Lettland, Litauen, Polen, Rumänien, Ungarn) oder die absolute Mehrheit verwendet (Belgien, Österreich, Bulgarien, Slowakei, Slowenien). Lediglich Tsche-

77 Benda, E., a.a. O., 127.

78 Tsebelis, G., a.a.O., 33 und 35. 
chien verlangt bei der abstrakten Normenkontrolle formal eine qualifizierte Mehrheit, nämlich neun von 15 Richtern. ${ }^{79}$

\section{Tabelle 8: Arenen, zentrale Akteure und Entscheidungsregeln}

\begin{tabular}{|c|c|c|c|c|c|c|c|c|c|}
\hline \multirow[b]{2}{*}{ Staat } & \multirow[b]{2}{*}{ Senate } & \multirow{2}{*}{$\begin{array}{l}\text { Kam- } \\
\text { mern }\end{array}$} & \multicolumn{3}{|c|}{ Gerichtspräsident } & \multirow{2}{*}{$\begin{array}{c}\text { Auswahl } \\
\text { Berichter- } \\
\text { statter }\end{array}$} & \multicolumn{3}{|c|}{ Abstimmungsregeln } \\
\hline & & & $\begin{array}{l}\text { Aus- } \\
\text { wahl }\end{array}$ & Amtszeit & $\begin{array}{c}\text { Wieder- } \\
\text { wahl }\end{array}$ & & $\begin{array}{c}\text { Mehr- } \\
\text { heit }\end{array}$ & $\begin{array}{c}\text { Patt- } \\
\text { situation }\end{array}$ & $\begin{array}{l}\text { Dis- } \\
\text { sent }\end{array}$ \\
\hline Belgien & nein $^{\mathrm{a}}$ & $1 \times 7$ & Gericht & $1 \mathrm{Jahr}$ & $\mathrm{ja}$ & Präsident & absolute $^{\mathrm{a}}$ & Präsident & nein \\
\hline $\begin{array}{l}\text { Deutsch- } \\
\text { land }\end{array}$ & $2 \times 8$ & $3 \times 5$ & $\begin{array}{l}\text { Wahl- } \\
\text { organ }\end{array}$ & Amtszeit & nein & $\begin{array}{l}\text { Plenum/ } \\
\text { Senat }\end{array}$ & einfache & $\begin{array}{l}\text { Zurück- } \\
\text { weisung }\end{array}$ & ja \\
\hline $\begin{array}{l}\text { Frank- } \\
\text { reich }\end{array}$ & nein & nein & $\begin{array}{l}\text { Wahl- } \\
\text { organ }\end{array}$ & Amtszeit & nein & Präsident & einfache & Präsident & nein \\
\hline Italien & nein & nein & Gericht & 3 Jahre & ja & Präsident & einfache & Präsident & nein \\
\hline $\begin{array}{l}\text { Öster- } \\
\text { reich }\end{array}$ & nein & $1 \times 5$ & $\begin{array}{l}\text { Wahl- } \\
\text { organ }\end{array}$ & Amtszeit & nein & Präsident ${ }^{b}$ & absolute & Präsident & nein \\
\hline Portugal & $3 \times 5^{\mathrm{c}}$ & nein & Gericht & 4,5 Jahre & $\mathrm{ja}$ & Los & einfache & Präsident & ja \\
\hline Spanien & $2 \times 6^{c}$ & $4 \times 3$ & Gericht & 3 Jahre & ja & Plenum & einfache & Präsident & ja \\
\hline $\begin{array}{l}\text { Bulga- } \\
\text { rien }\end{array}$ & nein & nein & Gericht & 3 Jahre & ja & Präsident & absolute & $\begin{array}{l}\text { Zurück- } \\
\text { weisung }\end{array}$ & ja \\
\hline Estland & nein $^{d}$ & nein & $\begin{array}{l}\text { Wahl- } \\
\text { organ }\end{array}$ & Amtszeit & nein & Plenum & einfache & Präsident & ja \\
\hline Lettland & nein & $3 \mathrm{er}$ & Gericht & 3 Jahre & ja & Präsident & einfache & $\begin{array}{l}\text { Zurück- } \\
\text { weisung }\end{array}$ & ja \\
\hline Litauen & nein & nein & $\begin{array}{l}\text { Wahl- } \\
\text { organ }\end{array}$ & Amtszeit & nein & Präsident & einfache & Präsident & nein \\
\hline Polen & nein & $\begin{array}{l}3 \mathrm{er} / \\
5 \mathrm{er}\end{array}$ & Gericht & Amtszeit & nein & Präsident & einfache & Präsident & ja \\
\hline $\begin{array}{l}\text { Rumä- } \\
\text { nien }\end{array}$ & nein & nein & Gericht & 3 Jahre & $\mathrm{ja}$ & Präsident & einfache & Präsident & ja \\
\hline $\begin{array}{l}\text { Slowa- } \\
\text { kei }\end{array}$ & nein & $4 \times 3^{e}$ & $\begin{array}{l}\text { Wahl- } \\
\text { organ }\end{array}$ & Amtszeit & nein & Los & absolute & $\begin{array}{l}\text { Zurück- } \\
\text { weisung }\end{array}$ & ja \\
\hline $\begin{array}{l}\text { Slowe- } \\
\text { nien }\end{array}$ & nein & $3 \times 3$ & Gericht & 3 Jahre & $\mathrm{ja}$ & $\begin{array}{c}\text { Alphabe- } \\
\text { tisch }\end{array}$ & absolute & - & ja \\
\hline $\begin{array}{l}\text { Tsche- } \\
\text { chien }\end{array}$ & nein & $4 \times 3$ & $\begin{array}{l}\text { Wahl- } \\
\text { organ }\end{array}$ & Amtszeit & $\mathrm{ja}$ & Präsident & $\begin{array}{l}\text { qualifi- } \\
\text { zierte }\end{array}$ & Präsident & ja \\
\hline Ungarn & nein & $3 \mathrm{er}$ & Gericht & 3 Jahre & ja & Präsident & einfache & Präsident & ja \\
\hline
\end{tabular}

Quelle: Eigene Darstellung

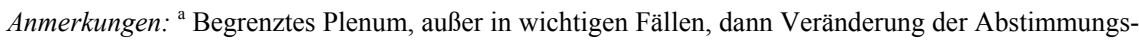
regel von einfacher zu absoluter Mehrheit. ${ }^{\mathrm{b}}$ Permanente Berichterstatter für 3 Jahre gewählt durch Plenum, Anzahl durch Gericht frei bestimmt, in letzten Jahren durchschnittlich 9. ' Plenumsvorbehalt bei Normenkontrollentscheidungen (Spanien) bzw. abstrakten Normenkontrollen (Portugal). ${ }^{\mathrm{d}}$ Der gesamte Supreme Court gliedert sich in 4 Kammern, u. a. die hier betrachtete Verfassungskammer, die in Panels unterschiedlicher Größe tagt. ${ }^{\mathrm{e}}$ Zur Zeit sind nur 11 Richter gewählt, Wahlverfahren noch laufend; daher ist auch die Anzahl der Senate unklar, aber höchstens 4 insgesamt.

79 Venice Commission: Bulletin on Constitutional Case-Law, a. a. O., 41. 
Kommt es bei der Abstimmung zu einem Patt, hat zumeist der Präsident das entscheidende Votum. Am deutlichsten wird dies in Österreich, wo der Präsident nur im Falle eines Patts abstimmt. ${ }^{80}$ Bis zur Einrichtung der mittel- und osteuropäischen Gerichte kannte nur das Bundesverfassungsgericht das Prinzip in dubio pro lege. Bei Stimmengleichheit kann also kein Verstoß gegen die Verfassung festgestellt werden ( $\$ 15$ BVerfGG). Diese Regelung wird nun auch von Bulgarien, Lettland und der Slowakei angewendet. Aufgrund der geraden Richteranzahl in Deutschland bedeutet dies, dass zur Annullierung eines Gesetzes eine 5:3 Mehrheit notwendig ist, was de facto eine qualifizierte Mehrheit darstellt. Slowenien wiederum kennt keine spezielle Regelung wegen des Erfordernisses der absoluten Mehrheit bei ungerader Richterzahl. Der wesentliche Unterschied dürfte letztlich zwischen der qualifizierten Mehrheit in Tschechien auf der einen und den einfachen und absoluten Mehrheiten auf der anderen Seite liegen, da bei schriftlichen Verfahren die Richter immer die Möglichkeit haben, ihre Stimme abzugeben. In einigen Ländern müssen die Richter sogar abstimmen: In Bulgarien ist jeder Richter zur Stimmabgabe verpflichtet und eine Enthaltung nicht zulässig. ${ }^{81}$

Mit Ausnahme Portugals wird das individuelle Abstimmungsverhalten der Richter nicht bekannt gegeben. Nur wenige Gerichte, wie das Bundesverfassungsgericht, veröffentlichen das kollektive Abstimmungsergebnis. In einigen Staaten ist dies sogar explizit verboten (z.B. Frankreich). Einen Einblick in individuelles Abstimmungsverhalten bieten Sondervoten. Stimmt ein Richter nicht mit der Mehrheit überein, kann er so seine Meinung veröffentlichen. Diese Möglichkeit besteht in den meisten Ländern mit Ausnahme Belgiens, Frankreichs, Italiens, Österreichs und Litauens.

\section{Fazit}

Die Verfassungsgerichte der EU-Staaten sind hinsichtlich ihrer institutionellen Struktur erstaunlich heterogen. Dies zeigt sich anhand der Verfahren zur Richterwahl, die darüber entscheiden, welche Präferenzen und juristischen Qualifikationen in das Gericht gelangen. Es betrifft die politische Isolation des Gerichtes, die den Richtern den nötigen Handlungsfreiraum gibt, ihre Entscheidungen unabhängig von kurzfristigen politischen Erwägungen zu treffen. Unterschiedlich 
geregelt ist auch der Zugang zum Verfassungsgericht durch politische und private Akteure, die nicht nur die Arbeitslast des Gerichts beeinflussen, sondern auch dessen Freiräume, wichtige Themen zu regeln. Schließlich variiert die interne Organisation des Gerichtes, die steuert, welche im Gericht vorhandenen Positionen eher zum Tragen kommen.

Im Gegensatz zur Forschung zum US Supreme Court, die diese institutionellen Regeln sehr detailliert analysiert hat, haben sich Untersuchungen zu europäischen Verfassungsgerichten vor allem auf das Verfahren der abstrakten Normenkontrolle, das Bundesverfassungsgericht und den Conseil constitutionnel sowie die allgemeine Hypothese der zunehmenden Justizialisierung konzentriert; die entsprechenden Ergebnisse erweisen sich als relativ uneinheitlich. ${ }^{82}$ Doch gerade die Verfassungsgerichte in Deutschland und Frankreich sind aus institutioneller Sicht am unterschiedlichsten, ${ }^{83}$ und so können auch die heterogenen Befunde nicht zwangsläufig verwundern.

Die hier vorgestellte Übersicht legt nahe, die politikwissenschaftliche Forschung - und hier insbesondere die vergleichende Analyse - zu den Verfassungsgerichten und ihrer Rolle im politischen System zu intensivieren und dabei einen stärkeren Fokus auf das Verständnis der verschiedenen institutionellen Arrangements zu legen. Dieses Vorgehen erlaubt nicht zuletzt eine genauere Antwort auf die Frage, welche Regelungen die viel beschworene „Verrechtlichung" der Politik fördern und welche sie bremsen.

Christoph Hönnige

82 Vanberg, G.: Abstract Judicial Review, Legislative Bargaining, and Policy Compromise, in: Journal of Theoretical Politics, 10/3 (1998), 299-326; Stone Sweet, A.: A Comment on Vanberg. Rules, Dispute Resolution, and Strategic Behavior, in: Journal of Theoretical Politics, 10/3 (1998), 327-338; ders.: Constitutional Courts, a. a. O.; Hönnige, C.: Verfassungsgericht, Regierung und Opposition, a.a.O.

83 Hönnige, C.: Die mittlere Sprosse der Leiter: Fallauswahl in Forschungsdesigns mit kleiner Fallzahl, in: Gschwend, T./Schimmelfennig, F. (Hg.): Forschungsdesign in der Politikwissenschaft, Frankfurt/New York, 2007, 223-250. 